\title{
Percepción de los Residentes de Medicina Familiar y Comunitaria sobre el programa formativo de la Especialidad
}

\author{
Francisco Javier Lucas Pérez-Romero ${ }^{a}$, Patricia Chacón Caso ${ }^{a}$, \\ Verónica Casado Vicente ${ }^{b}$, Francisco Pablo Cerezuela ${ }^{\mathrm{c}}$
}

\author{
a Ex vocal de residentes \\ de la Comisión Nacional \\ de Medicina Familiar y \\ Comunitaria. \\ ${ }^{b}$ Presidenta de la Comisión \\ Nacional de Medicina Familiar \\ y Comunitaria. \\ c Vicepresidente de la Comisión \\ Nacional de Medicina Familiar y \\ Comunitaria.
}

Correspondencia: Francisco Javier Lucas Pérez-Romero, Avenida José Prat $n^{\circ} 14$, Bloque 2, Escalera 3, $6^{\circ} \mathrm{F} 2$, 02006 - Albacete.

Correo electrónico: javi_lucas@hotmail.com.

Recibido el 13 de octubre de 2011.

Aceptado para su publicación el 16 de enero de 2012.

\section{RESUMEN}

Objetivo. Conocer la opinión de las dos primeras promociones de residentes de Medicina familiar y Comunitaria (MFyC) sobre el Tercer Programa Oficial de la Especialidad y evidenciar fortalezas y debilidades.

Diseño. Estudio observacional descriptivo transversal.

Emplazamiento. Todas las Unidades Docentes de MFyC de España.

Participantes. Residentes que finalizaron la residencia de MFyC en mayo de 2008 y 2009.

Mediciones principales. Sociodemográficas, grado de satisfacción con la formación y con los docentes, percepción de presión asistencial, publicaciones, distribución de las rotaciones y uso del libro del residente.

Resultados. Se recibieron 450 encuestas (201 en 2008 y 249 en 2009). La edad media fue de 31,46 años $\pm 4,82 \mathrm{DE}(2008)$ y $32 \pm 4,93 \mathrm{DE}$ (2009). El $68,7 \%$ fueron mujeres en 2008 y el $76 \%$ en 2009. La puntuación media del grado de satisfacción con la residencia fue de 7,45 puntos \pm $1,34 \mathrm{DE}(2008)$ y $7,29 \pm 1,29 \mathrm{DE}(2009)$, la valoración del tutor $8,07 \pm 1,79 \mathrm{DE}(2008)$ y $7,90 \pm$ $2,11 \mathrm{DE}$ (2009). La puntuación en la capacidad docente fue de $8,22 \pm 1,82 \mathrm{DE}$ y en competencia clínica de $8,67 \pm 1,27 \mathrm{DE}$. El número medio de consultas fue de 39,13 $\pm 9,38 \mathrm{DE}(2008)$ y de $38,14 \pm 10,28$ DE (2009) y el tiempo de formación en Atención Primaria (AP) en torno a 20 meses (rotación rural $70 \%$ y $64 \%$, respectivamente). El $82 \%$ había publicado en 2009 , frente al $55,7 \%$ en 2008. En torno al $60 \%$ había realizado actividades de Atención a la Familia y a la Comunidad. La valoración del libro del residente obtuvo una puntuación media de 5,26 $\pm 2,16$ DE (2008) y 4,53 $\pm 2,40$ DE (2009)

Conclusiones. El grado de satisfacción con la residencia fue notable. La figura mejor valorada es el tutor. El tiempo de rotación en AP se ajusta al programa. Las áreas de mejora percibidas: implementación del libro del residente, presión asistencial, rotación rural, atención a la familia y a la comunidad e investigación.

Palabras clave. Medicina Familiar y Comunitaria, Especialización.

\section{ABSTRACT}

Family and Community Medicine Residents evaluate their training program

Objectives. To elicit the opinion of the first two graduating classes of Family and Community Medicine residents (FCM) about the Third Official Program of the specialty and to discover its strengths and weaknesses.

Design. Observational, descriptive cross-sectional study.

Setting. All FCM teaching departments in Spain.

Participants. Graduates who finished residency training in FCM in May 2008 and 2009.

Main measures. Sociodemographic variables, degree of satisfaction with training and with teachers, perception of patient load, publications, distribution of rotations and use of residency book.

Results. 450 questionnaires were received (201 in 2008 and 249 in 2009). Average age of participants was $31.46 \pm 4.82$ (2008) and $32 \pm 4.93$ (2009). In 2008, $68.7 \%$ of respondents were women, and in 2009, $76 \%$. Degree of satisfaction with residency was: $7.45 \pm 1.34$ (2008) and $7.29 \pm 1.29$ (2009). Evaluation of instructor, 8.07 \pm 1.79 (2008) and $7.90 \pm 2.11$ (2009), with a score of $8.22 \pm 1.82$ in teaching capability and $8.67 \pm 1.27$ in clinical competence. Average number of practices visited was $39.13 \pm 9.38$ (2008) and $38.14 \pm 10.28$ (2009). Training period in PC (Primary Care) was approximately 20 months (rural rotation $70 \%$ and $64 \%$ in 2008 and 2009 , respectively). $82 \%$ published research in 2009 compared to $55.7 \%$ in 2008 . Approximately $60 \%$ of residents had been actively involved in Family and Community Care. Evaluation of residency book was $5.26 \pm 2.16(2008)$ and $4.53 \pm 2.40$ (2009).

Conclusions. Degree of satisfaction with residency training was considerable. The most valued aspect of the program was the instructor. Rotation time in PC meets program requirements. Areas needing improvement: use of residency book, patient load, rural rotation, family and community care, and research.

Key Words: Family Practice, Specialization. 


\section{INTRODUCCIÓN}

Ante la necesidad de nuestro Sistema Nacional de Salud de formar médicos de familia cada vez más cualificados, con nuevas competencias y capacitados para trabajar con alto nivel de resolutividad en Atención Primaria y en otros entornos diferentes, el 3 de Mayo de 2005 se publicó el tercer Programa oficial de la especialidad de Medicina Familiar y Comunitaria (MFyC) ${ }^{1}$, que incorporaba grandes cambios sobre su estructura interna, configuración docente, metodologías, contenidos y entornos e itinerarios formativos, incrementándose a cuatro años de formación.

El nuevo Programa, hasta el momento, ha tenido una importante repercusión interna y externa ${ }^{2}$. En la externa se reconoce su impacto en la norma sobre la figura del tutor (RD 183/2008), en sus desarrollos autonómicos y en la estructura de los programas de otras especialidades. Ha influido en la definición y formulación de la troncalidad y del tronco médico. A nivel interno ha conllevado un nuevo libro del residente, único publicado en la página web del Ministerio de Sanidad, Política Social e Igualdad ${ }^{4}$. Ha originado dos resoluciones de gran trascendencia ${ }^{5,6}$, una sobre acreditación de las Unidades Docentes (UUDD) de MFyC, que define los criterios de calidad de las mismas y de sus dispositivos, y otra sobre la incorporación de una rotación en atención primaria en los programas formativos de 18 especialidades médicas. Ha propiciado el Plan de Auditorias Docentes y el proceso de acreditación y reacreditación de las 97 UUDD (170 sectores), 214 hospitales y 856 Centros de Salud (CS) docentes.

Este proceso está permitiendo poner de manifiesto fortalezas como una red muy amplia y consolidada, una estructura docente comprometida, un programa instaurado y en pleno desarrollo, unos cronogramas mayoritariamente adaptados al programa, un muy buen equipamiento docente en un muy amplio número de CS docentes, un buen nivel de actividad docente, una mejora importante de la actividad investigadora, unos buenos planes de evaluación global y un ajuste progresivo de ratios poblacionales de habitantes por médico de familia en varias comunidades autónomas ${ }^{7}$.

Pero también se evidencian áreas de mejora, que incluyen adecuar las condiciones asistenciales de los recursos docentes y aumentar el tiempo de dedicación de jefes de estudios y técnicos de salud a las UUDD, seguir consolidando áreas competenciales, potenciando la investigación en algunas UUDD y profundizando en las herramientas para la actividad formativa y evaluativa ${ }^{7}$. Todos estos análisis serían insuficientes sin la opinión de los protagonistas y por esta razón la Comisión Nacional (CN) de MFyC, a través de los vocales de los residentes, ha realizado durante dos años consecutivos una encuesta para valorar la opinión de la primera y segunda promoción de residentes de MFyC con su nuevo Programa.

Existen pocos resultados publicados en cuanto a encuestas globales de opinión de los residentes sobre la calidad de la formación especializada ${ }^{8}$. Al revisar la literatura encontramos varias publicaciones de otras especialidades que abarcan a todos los residentes de un mismo centro hospitalario o de varios centros ${ }^{9-20}$. No nos consta ninguna que evalúe la opinión de residentes de MFyC a nivel nacional, aunque encontramos algunas de otros países y con otros enfoques más específicos $^{21-22}$.

El objetivo principal de este estudio es conocer la opinión que tienen la primera y segunda promociones de Residentes de MFyC sobre los cambios fundamentales que generó la publicación de dicho Programa. Los objetivos secundarios consisten en evidenciar fortalezas y debilidades en el actual proceso formativo, así como la evolución de las mismas con el paso del tiempo.

\section{MATERIAL Y MÉTODOS}

Se trata de un estudio observacional descriptivo de tipo transversal, realizado mediante encuesta. En junio de 2008 vía correo electrónico se remitió desde la CN de MFyC una encuesta de opinión a todas las UUDD de España para su difusión a todos los Residentes de MFyC de su Unidad Docente.

El requisito para poder contestar la encuesta consistía en haber terminado la residencia de MFyC durante el mes anterior (pertenecer a la primera promoción de residentes de Medicina de Familia con el programa de cuatro años).

La encuesta constaba de datos generales (edad, sexo, unidad docente y comunidad autónoma del encuestado) y 36 preguntas (tablas 1-5), debiendo remitirse tras su cumplimentación a una dirección electrónica.

En mayo de 2009 se envió una encuesta de respuesta on-line, con el mismo encabezamiento y 


\begin{tabular}{l|cc}
\hline & 2008 & 2009 \\
\hline - $\quad$ Grado de satisfacción global con la residencia & $7,45 \pm 1,34$ & $7,29 \pm 1,29$ \\
- $\quad$ Grado de satisfacción con las rotaciones realizadas & $7,09 \pm 1,35$ & $7,11 \pm 1,57$ \\
- $\quad$ Grado de dedicación de su tutor en su labor de tutorización & $8,07 \pm 1,79$ & $7,90 \pm 2,11$ \\
- $\quad$ Grado de dedicación de su Coordinador-Jefe de Estudios en su labor de & $6,27 \pm 2,35$ & $6,63 \pm 2,35$ \\
planificación y supervisión de la formación & & $5,98 \pm 2,14$ \\
\hline
\end{tabular}

Tabla 1. Valoraciones de diferentes figuras y circunstancias de la residencia. Escala analógica (0-10).

\begin{tabular}{|c|c|c|}
\hline & 2008 & 2009 \\
\hline $\begin{array}{l}\text { - ¿Está dotado su Centro de Salud de todo el material necesario para llevar a } \\
\text { cabo todas las actividades asistenciales? }\end{array}$ & Si: $71,6 \%$ & Si: $67 \%$ \\
\hline $\begin{array}{l}\text { - ¿Se ofertan en su centro todas las competencias relacionadas con la } \\
\text { Atención al individuo que aparecen en el Programa de la Especialidad? }\end{array}$ & Si: $68,2 \%$ & Si: $82 \%$ \\
\hline - ¿ ¿Realizan actividades de Atención a la Familia en su Centro de Salud? & Si: $61,2 \%$ & Si: $59 \%$ \\
\hline - ¿ ¿Realizan actividades de Atención a la Comunidad en su Centro de Salud? & Si: $57,7 \%$ & Si: $69 \%$ \\
\hline $\begin{array}{l}\text { - ¿Tienen ordenador con conexión a Internet accesible al Residente en su } \\
\text { centro de salud? }\end{array}$ & Si: $77,6 \%$ & Si: $93 \%$ \\
\hline - ¿Cuál es el número medio de consultas de su tutor al día? & $39,13 \pm 9,38$ & $38,14 \pm 10,28$ \\
\hline $\begin{array}{l}\text { - ¿Cuántas horas de actividad asistencial en consultas o domicilios realiza al } \\
\text { día? }\end{array}$ & $6,27 \pm 2,32$ & $5,74 \pm 1,94$ \\
\hline
\end{tabular}

Tabla 2. Preguntas referentes a varios aspectos del Centro de Salud.

\begin{tabular}{|c|c|c|c|}
\hline & & 2008 & 2009 \\
\hline- & $\begin{array}{l}\text { ¿Figura como autor/coautor en alguna Comunicación o Publicación durante su } \\
\text { residencia? }\end{array}$ & Si: $55,7 \%$ & Si: $82 \%$ \\
\hline- & En caso afirmativo, Indique cuántas & $1,80 \pm 3,40$ & $4,65 \pm 9,39$ \\
\hline- & ¿Ha realizado Cursos de Doctorado durante su residencia? & Si: $56,7 \%$ & Si: $43 \%$ \\
\hline
\end{tabular}

Tabla 3. Preguntas referentes a aspectos formativos. 


\begin{tabular}{llc}
\hline & 2008 & 2009 \\
\hline - ¿Cuántos meses ha rotado por Medicina de Familia & $19,67 \pm 3,09$ & $\begin{array}{r}4,42 \pm 1,46\left(1^{\circ} \text { año) }\right. \\
\text { durante su Residencia? }\end{array}$ \\
- ¿Ha realizado rotación rural? & Si: $70,1 \%$ & Si: $64 \%$ \\
- ¿Ha realizado estancias electivas/rotatorio libre? & Si: $75,6 \%$ & Si: $73 \%$ \\
- ¿Ha realizado rotaciones externas? & Si: $39,3 \%$ & Si: $29 \%$ \\
- ¿Ha realizado al menos $\%$ & Si: $92 \%$ \\
$\quad$ de programa teórico práctico obligatorio? & Si: $95 \%$ & Si: $97 \%$ \\
\hline
\end{tabular}

Tabla 4. Preguntas referentes a diferentes aspectos de las rotaciones realizadas.

\begin{tabular}{|c|c|c|c|}
\hline & & 2008 & 2009 \\
\hline - & $\begin{array}{l}\text { ¿Cree que ha mejorado la formación del Residente con } \\
\text { el nuevo Programa? }\end{array}$ & Si: $72,6 \%$ & Si: $73 \%$ \\
\hline - & $\begin{array}{l}\text { ¿Con qué frecuencia ha utilizado el Libro del Residente } \\
\text { en Formación durante su Residencia? }\end{array}$ & Si: $59,7 \%$ & $\begin{array}{c}\text { Nunca: } 26 \% \text {, Semanalmente: } 1 \% \text {. } \\
\text { Una vez al mes: } 6 \% \text {, } \\
\text { Trimestralmente: } 19 \% \text {, Una vez al } \\
\text { año: } 28 \% \text {, Menos de una vez al } \\
\text { año: } 21 \% .\end{array}$ \\
\hline- & $\begin{array}{l}\text { Indique que valoración le merece el libro del Residente } \\
\text { como herramienta de reflexión formativa }\end{array}$ & $5,26 \pm 2,16$ & $4,53 \pm 2,60$ \\
\hline
\end{tabular}

Tabla 5. Preguntas referentes a varios aspectos del nuevo programa de la especialidad.

\begin{tabular}{l|cc}
\hline \multicolumn{1}{c|}{ Comunidad Autónoma } & $\mathbf{2 0 0 8}$ & $\mathbf{2 0 0 9}$ \\
\hline Andalucía & $15,9 \%$ & $15,9 \%$ \\
Aragón & $1,5 \%$ & $5,3 \%$ \\
Asturias & $2 \%$ & $5,7 \%$ \\
Baleares & $2,5 \%$ & $4,9 \%$ \\
Canarias & $4,5 \%$ & $1,6 \%$ \\
Cantabria & $1,5 \%$ & $2 \%$ \\
Castilla-La Mancha & $7,5 \%$ & $6,9 \%$ \\
Castilla León & $6 \%$ & $7,7 \%$ \\
Cataluña & $18,9 \%$ & $26,6 \%$ \\
Comunidad Valenciana & $5,5 \%$ & $1,2 \%$ \\
Extremadura & $2,5 \%$ & $1,6 \%$ \\
Galicia & $8 \%$ & $5,3 \%$ \\
La Rioja & $1,5 \%$ & $0,8 \%$ \\
Madrid & $10,9 \%$ & $10,6 \%$ \\
Murcia & $5 \%$ & $0 \%$ \\
Navarra & $0,5 \%$ & $2 \%$ \\
País Vasco & $2 \%$ & $3,2 \%$ \\
No especificado & $4 \%$ & $0 \%$ \\
& & \\
\hline
\end{tabular}

Tabla 6. Porcentaje de respuestas recibidas según comunidad autónoma. 
un aumento de preguntas, hasta 62 , con el objetivo de mejorar la redacción y preguntar sobre más temas que la primera no recogía (tablas 1-5).

Todos los datos fueron almacenados en una base de datos Access y procesados mediante el paquete estadístico SPSS v.12. Los resultados cuantitativos se expresan en forma de media aritmética, desviación estándar (DE) e intervalo de confianza al 95\% (IC 95\%), así como mediana y valores mínimo y máximo. Los resultados cualitativos se expresan en forma de porcentaje e intervalo de confianza al 95\% (IC 95\%). Se empleó el test no paramétrico de Mann-Whitney para comprobar la homogeneidad de la muestra. El nivel de significación utilizado fue el de $p<0,05$.

\section{RESULTADOS}

Se recibieron 201 encuestas de los residentes que finalizaron en 2008 y 249 de los que lo hicieron en 2009. No se rechazó ninguna de ellas por mala cumplimentación en ninguno de los dos años. En 2008 contestaron residentes de todas las Comunidades Autónomas, excepto Ceuta y Melilla, que en esos momentos no contaban con residentes. El año siguiente no se recibieron tampoco de la Región de Murcia (tabla 6). La edad media de los encuestados el primer año fue de 31,46 años $\pm 4,82$ $\mathrm{DE}$, siendo varones el $31,3 \%$ y mujeres el $68,7 \%$. En 2009 los resultados fueron 32 años \pm 4,93 DE, con un $76 \%$ de mujeres.

En 2008 el grado de satisfacción con la residencia, en una escala analógica de 0 a 10 , fue de 7,45 puntos $\pm 1,34 \mathrm{DE}$, puntuando por debajo de 5 tan solo el $3 \%$ de los encuestados. Muy parecida valoración se otorgó a las rotaciones realizadas $(7,09 \pm 1,35$ $D E)$. Lo mejor valorado por los residentes fue su tutor $(8,07 \pm 1,79 \mathrm{DE})$, siendo sensiblemente peor valorada la figura del jefe de estudios en su labor de planificación y supervisión de su formación $(6,27 \pm 2,35 \mathrm{DE})$, así como la calidad de los cursos ofertados por la unidad docente $(6,23 \pm 1,87 \mathrm{DE})$. Las 200 horas lectivas teórico-prácticas fueron realizadas por el $95 \%$.

La segunda promoción valoró su satisfacción con la residencia con una puntuación de 7,29 $\pm 1,29 \mathrm{DE}$, puntuando por debajo de 5 el $3,6 \%$ de los mismos. Las rotaciones realizadas fueron calificadas con $7,11 \pm 1,57 \mathrm{DE}$. La figura del tutor obtuvo $8,22 \pm$ $1,82 \mathrm{DE}$ en cuanto a capacidad docente y 8,67 $\pm 1,27 \mathrm{DE}$ en cuanto a competencia clínica. Su dedicación a tutorizar alcanzó 7,90 $\pm 2,11 \mathrm{DE}$. El jefe de estudios fue valorado con 6,63 $\pm 2,35 \mathrm{DE}$. La calidad de los cursos obtuvo una valoración de $5,98 \pm 2,14 \mathrm{DE}$. El $92 \%$ de los encuestados habían realizado al menos 200 horas teórico-prácticas.

Una de las grandes novedades del nuevo Programa era el libro del residente, que usó el $59,7 \%$ de los encuestados en 2008 frente al $76 \%$ en 2009 , dándole una calificación como herramienta de reflexión formativa de 5,26 $\pm 2,16 \mathrm{DE}$ los primeros y de 4,53 $\pm 2,60 \mathrm{DE}$ los segundos. En 2009 se preguntó también la frecuencia de su uso. Lo utilizaba semanalmente el $1 \%$, una vez al mes el $8 \%$, trimestralmente el $25 \%$, una vez al año el $37 \%$ y menos de una vez al año el $28 \%$. Entre los que no lo usaban alegó no tenerlo el $27 \%$, no ser útil para el $37 \%$, no usarse en su Unidad Docente el $26 \%$ y no tenía tiempo suficiente para su uso el $10 \%$.

En 2008 consideraba que sus centros estaban bien dotados de medios el $71 \%$, frente al $67 \%$ en 2009 . Un $20 \%$ de los centros no disponían de biblioteca el primer año, frente a un $8 \%$ que declaró no tenerla en su centro de salud en 2009 . Un $22 \%$ de los residentes no tenía un ordenador conectado a Internet fuera de la consulta a su disposición en 2008, frente al 7\% en 2009.

En el análisis del número medio de consultas por profesional y día, los residentes encuestados afirmaron en 2008 que el $58 \%$ de los médicos atendían diariamente a más de 35 pacientes, siendo la media de pacientes diarios de 39,13 \pm 9,38 DE. En 2009 este dato fue del $53 \%$, con una media de 38,14 \pm 10,28 DE. En 2009 también se les preguntó por el número óptimo según ellos de consultas diarias, siendo 20-29 el valor más seleccionado (45\%) seguido de $30-39$ (37\%). La jornada asistencial media de estos profesionales era de 6,27 horas diarias $\pm 2,32$ DE en 2008 y de $5,74 \pm 1,94$ DE en 2009. El $62 \%$ de los residentes afirmó trabajar más de 5,5 horas diarias atendiendo a pacientes en 2008, en 2009 más de 6 horas el $61 \%$ de los encuestados.

En 2008 afirmaron pasar la consulta una media de 5,51 meses $\pm 5,36 \mathrm{DE}$ sin supervisión directa por parte de su tutor. En 2009 fue reformulada ésta pregunta, observándose que el $77 \%$ pasaba consulta de manera autónoma sin la supervisión directa de su tutor al menos dos meses completos y siendo la media de 6,45 meses $\pm 3,99 \mathrm{DE}$. El $66 \%$ contestó que había pasado la consulta de otros cupos diferentes al de su tutor, pero tratándose en la mayoría de días sueltos. 
Entre los residentes encuestados había realizado actividades comunitarias el $58 \%$ y atención a la familia el $61 \%$ en 2008, frente al $69 \%$ y $59 \%$ respectivamente en 2009. En 2009 también se preguntó sobre si se ofertaban en su centro de salud todas las competencias relacionadas con la atención al individuo, contestando afirmativamente el $82 \%$ y siendo la cirugía menor la menos desarrollada.

En 2008 el número medio de publicaciones durante la residencia fue de 1,80 $\pm 3,40 \mathrm{DE}$. El 44,3\% de los encuestados no publicó nada durante su periodo formativo. En 2009 fueron autores o coautores de alguna publicación o comunicación el $82 \%$, y entre los que publicaron el número medio fue 4,65 \pm 9,39 DE. El $68 \%$ de los residentes afirmaron participar en alguna línea de investigación.

Los que finalizaron en 2008 declararon haber rotado 19,67 meses $\pm 3,09 \mathrm{DE}$ en AP, realizando el $27,57 \%$ de sus guardias también en AP. El $70,1 \%$ había realizado una rotación en el medio rural, el $75,6 \%$ había realizado algún mes de estancia electiva y algo más de un tercio de los encuestados $(39,3 \%)$ una rotación externa. En 2009 la duración media de rotación en AP durante su R1 fue de 4,42 meses $\pm 1,46 \mathrm{DE}$ y de 16,34 $\pm 4,06 \mathrm{DE}$ en el periodo comprendido entre R2 y R4. El número medio de guardias realizadas mensualmente durante su residencia fue de 5,06 $\pm 1,25 \mathrm{DE}$.

En 2008 realizaron durante la residencia cursos de doctorado el $56,7 \%$ de los residentes. El 20,9\% refirió haber tenido dificultades para realizar estos cursos. En 2009 el 43\% realizó los cursos de doctorado, finalizando la tesis el $6 \%$ antes de terminar la residencia. Un $57 \%$ de estas tesis estaban relacionadas con la medicina de familia. El director de su tesis era médico de familia en el $12 \%$ de las ocasiones.

En 2008 el 87,1\% de los residentes opinaba que había cumplido con el Programa de formación, encontrándose capacitados para el desempeño laboral prácticamente la totalidad $(99,5 \%)$ de los encuestados. En 2009 esta pregunta se formuló de manera diferente, puntuando en una escala analógica de 0-10 su capacitación. La puntuación media obtenida fue de 7,66 $\pm 1,13 \mathrm{DE}$. El $98,5 \%$ de los mismos se otorgó una calificación por encima de 5 . El 18,9\% se consideró excelente (por encima de 9). Finalmente, en 2008 el 73\% consideró que el nuevo Programa mejoraba la formación con respecto al anterior. Un año más tarde esta proporción fue del $74 \%$.

\section{DISCUSIÓN}

Los datos obtenidos en el presente estudio son meramente orientativos, ya que para un universo de 1.433 residentes que acabaron la residencia en mayo de 2008 y 1.312 que lo hicieron en mayo de 2009 , con un intervalo de confianza del $95 \%$, un error del $5 \%$ y una prevalencia del $50 \%$ hubiésemos necesitado un tamaño muestral de 304 para el año 2008 y 298 para 2009. Hemos obtenido únicamente 201 encuestas en 2008 y 249 en 2009 (14\% y 19\% de los residentes respectivamente), con un sesgo de representación y probablemente de distribución. Para minorar el sesgo de representación, hemos juntado dos promociones (un universo de 2.745 precisa una muestra de 338 ), habiendo obtenido 450 encuestas contestadas.

También son evidentes los posibles sesgos que pudiera tener el presente estudio por la mala difusión que podría haber tenido la encuesta, los diferentes grados de satisfacción y de implicación y la subjetividad de la interpretación personal de determinadas preguntas de redacción confusa.

A favor de los resultados obtenidos debemos considerar la homogeneidad de las respuestas obtenidas, siempre con la salvedad de las pocas encuestas recibidas desde algunas UUDD, y la similitud de la mayoría de los datos en las encuestas de los dos años.

Estos resultados permiten a la CN de MFyC, junto a la información que está siendo analizada en el proceso de acreditación y reacreditación de las UUDD y de sus dispositivos, poder evidenciar fortalezas y debilidades sobre las que se debe seguir trabajando.

Así pues, el hecho de que todavía un tercio de los residentes declare no rotar en el medio rural nos indica la necesidad de potenciar la rotación rural prevista de 3 meses durante el periodo de R2 en el Programa formativo de la especialidad, debido a las connotaciones específicas que tienen las actividades que realizan los médicos de familia en este medio.

Un aspecto negativo, ya evidenciado en otros estudios $^{7,23-26}$, es la elevada presión asistencial que soporta el médico de familia, con una media en torno a 40 pacientes diarios y un elevado número de horas dedicadas a la consulta durante la jornada asistencial. 
Estos podrían ser los motivos, junto con la formación propia de los tutores, que explican que más de un tercio de nuestros residentes no realice actividades de Atención a la Familia y a la Comunidad, indicando la necesidad de disminuir los cupos y de potenciar dicha formación y sus actividades al ser consideradas por el Programa competencias específicas del perfil de un especialista en MFyC.

El ítem con peores resultados fue el libro del especialista en formación, que aparte de ser usado con poca frecuencia ha tenido una pobre aceptación. Esta crítica y el valor intrínseco de la herramienta instan a trabajar sobre el mismo.

Si bien los resultados obtenidos han mejorado de un año a otro, deberíamos tener en cuenta el fomento de la investigación entre los residentes de nuestra especialidad y la calidad de los cursos.

Como datos positivos destacamos la buena valoración que le dan los residentes a la figura del tutor (elemento imprescindible en la formación) y al grado de satisfacción global con la residencia, siendo ambos resultados superiores a otras encuestas de satisfacción ${ }^{8,10,12}$. Estos dos datos, según los resultados obtenidos en otras encuestas de opinión de residentes, parecen ir muy emparejados ${ }^{11}$.

También son datos positivos el elevado cumplimiento del nuevo Programa y el adecuado porcentaje de rotación en medicina de familia en CS y entornos próximos a la Atención Primaria en la mayoría de los casos.

Consideramos que estas encuestas deben mantenerse y debe intentarse mejorar el porcentaje de respuestas adelantando la encuesta en el tiempo e incrementando la implicación de los jefes de estudio en su difusión, así como la de los residentes en su cumplimentación. Valoramos globalmente los resultados obtenidos de manera positiva, ya que la puesta en marcha de un programa nuevo, más extenso y complejo, no está exenta de dificultades para las primeras promociones.

\section{AGRADECIMIENTOS}

A los residentes de la especialidad de Medicina Familiar y Comunitaria que han cumplimentado la encuesta, a los jefes de estudio de las Unidades Docentes que la han difundido y a los miembros de la Comisión Nacional por sus sugerencias.

\section{BIBLIOGRAFÍA}

1. Comisión Nacional de Medicina Familiar y Comunitaria. Programa Oficial de la Especialidad de Medicina Familiar y Comunitaria. Madrid: Ministerio de Sanidad y Consumo; 2005. Disponible en: http://www.msps.es/profesionales/ formacion/docs/mediFamiliar07.pdf.

2. Chacón Caso P, Lucas Pérez-Romero J, Casado Vicente V. Los primeros R4 de Medicina Familiar y Comunitaria. AMF. $2009 ; 5(11): 614-5$.

3. Boletín Oficial del Estado. Real Decreto 183/2008 de 21 de febrero, por el que se determinan y clasifican las especialidades en ciencias de la salud y se desarrollan determinados aspectos del sistema de formación sanitaria especializada. BOE número 45 de 21/02/2008.

4. Comisión Nacional de Medicina Familiar y Comunitaria. Libro del Residente de la Especialidad de Medicina Familiar y Comunitaria. Madrid: Ministerio de Sanidad y Consumo; 2007. Disponible en: http://www.msc.es/profesionales/ formacion/docs/libroResidenteMedFamiliar.pdf.

5. Resolución conjunta de los Ministerios de Sanidad y Consumo y de Educación y Ciencia, por la que se establecen los Requisitos Generales de Acreditación de las Unidades Docentes para la formación de Médicos Especialistas en Medicina Familiar y Comunitaria. Resolución de 9 de junio de 2005.

6. Resolución de 15 de junio de 2006, de la Dirección General de Recursos Humanos y servicios económicospresupuestarios, del Ministerio de Sanidad y Consumo sobre la incorporación progresiva de una rotación en atención primaria de salud, en los programas formativos de determinadas especialidades sanitarias

7. Casado V, Pablo F. La acreditación de las UUDD de MFyC de España: estado de situación. Jornadas de Jefes de Estudio de las UUDD de Medicina Familiar y Comunitaria de España. Ministerio de Sanidad y Política Social. Madrid. 2 de Marzo de 2010.

8. Moro J, Tejedor JM, Zancajo JL. La calidad de la formación especializada a través de la encuesta de opinión de residentes Rev Calidad Asistencial. 2006; 21(2):82-6.

9. Pujol R, Nogueras A. La formación de los médicos residentes de Medicina Interna. A propósito de una encuesta de opinión. Med Clin (Barc). 2002;118:545-50.

10. Ríos A, Sánchez F, González L, Guerrero M. Factores de insatisfacción de los médicos internos residentes. Med Clin (Barc). 2003; 121:634-6.

11. Ríos A, Sánchez F, González L, Guerrero M. Importancia del tutor de la especialidad en la satisfacción y formación de los médicos internos residentes. Nefrología. 2005; 25(3):246-9.

12. Blasco Casares FJ. Insatisfacción de los médicos internos residentes. Med Clin (Barc). 2004;123(4):157.

13. Ríos Zambudio A, Sánchez Gascón F, González Moro L, Guerrero Fernández M. Research training during medical residency (MIR). Satisfaction questionnaire. Rev Esp Enferm Dig 2004; 96:695-704.

14. Pijoán Jl, Urkaregi A, Morán JM. Evaluación por los médicos internos residentes de la formación recibida en los servicios hospitalarios: una herramienta de monitorización. Gac Sanit. 2001; 15:432-40.

15. Fernández Llamazares J, Julián JF, Hidalgo F, García F, Moreno P, Vega JL. Encuesta a residentes MIR sobre su satisfacción tras obtener una plaza hospitalaria. Med Clin (Barc). 1997; 109:615-8.

16. Zambrana García JO, Díez García F, Martín Escalante MD, Rivera Cívico F, Delgado Fernández M, Cruz Caparrós G. ¿Qué opinan los residentes de Medicina Interna 
de Andalucía de su especialidad? Resultados de una encuesta. An Med Interna (Madrid). 1999; 16:484-7.

17. Rodríguez González FJ, Puente Gutiérrez J, Chicano Gallardo M, Monrobel Lancho A, López Peñas D, Bernal Blanco E. Formación MIR en aparato digestivo. El punto de vista de los residentes. Gastroenterol Hepatol. 2000; 23:367-73.

18. Pinsach Elias L, Valero Millian J, Blasco Casares FJ. La especialización en Urología. Parte III. La opinión de los médicos internos residentes. Actas Urol Esp. 1996; 20:11738.

19. Rosell A, Miravitlles M, Roger N, Jolis R, Llunell A, Morell F. Los residentes de neumología formados en Cataluña (1989-1993). Med Clin (Barc). 1996; 106:653-5.

20. Campbell J, Prochazka AV, Yamashita T, Gopal R. Predictors of persistent burnout in internal medicine residents: a prospective cohort study. Acad Med. 2010; 85(10):1630-4.

21. Cárdenas-Ayón,E, Cabrera-Pivaral, CE, ViniegraVelázquez,L, González-Reyes,HF, Luce-González,EG, Marco Antonio Ortiz-López MA. Satisfacción de los residentes de medicina familiar con el ambiente académico laboral. Rev Med Inst Mex Seguro Soc. 2010; 48(2):227-31.

22. McCray LW, Cronholm PF, Bogner HR, Gallo JJ, Neill RA. Resident physician burnout: is there hope? Fam Med. 2008; 40(9):626-32.

23. Prieto Albino L. Burnout en médicos de atención primaria de la provincia de Cáceres. Aten Primaria. 2002: 30(5): 294-302.

24. Sobrequés Soriano J, Cebriá Andreu J, Segura Bernal J, Rodríguez Muñoz C, García García M, Juncosa Font S. La satisfacción laboral y el desgaste profesional de los médicos de atención primaria. Aten Primaria. 2003; 31(4):227-33.

25. Cortés Rubio JA, Martín Fernández J, Morente Páez M, Caboblanco Muñoz M, Garijo Cobo J, Rodríguez Balo A. Clima laboral en atención primaria: ¿qué hay que mejorar? Aten Primaria. 2003; 32:288-95.

26. Freeborn DK. Satisfaction and Well-Being of Primary Care Providers in Managed Care. Eval Health Prof. 2002; 25(2):239-54 